\title{
Vibrating wire scanner: First experimental results on the injector beam of the Yerevan synchrotron
}

\author{
S. G. Arutunian, N. M. Dobrovolski, M. R. Mailian, and I. E. Vasiniuk \\ Yerevan Physics Institute, 2 Alikhanian Brothers Street, 375036 Yerevan, Armenia
}

(Received 9 August 2002; published 11 April 2003)

\begin{abstract}
This paper presents the first experimental results of the transverse profile measurements using a vibrating wire scanner at the Yerevan synchrotron injector electron beam ( $6 \mathrm{nA}$ after collimation). The advantage of this novel technique is that there is no need for secondary radiation or particle detectors. The local beam intensity is measured using the natural oscillation frequency of the wire, which is a function of the wire temperature.
\end{abstract}

DOI: 10.1103/PhysRevSTAB.6.042801

PACS numbers: 29.27.Fh

\section{INTRODUCTION}

We propose to use a vibrating wire scanner (VWS) to measure charged particle beam transverse profiles [1]. The advantage of this technique is that there is no need for additional auxiliary beam lines to detect secondary radiation or particles as is common for traditional wire scanners.

The interaction of the beam with the wire mainly causes heating of the wire. For instance, it is estimated that $35.5 \mathrm{keV}$ of the $100 \mathrm{MeV}$ proton beam energy is deposited for heating the wire, and only $0.67 \mathrm{keV}$ of energy is transformed into secondary radiation or particles [2]. Thus we expect that the frequency of natural oscillations of the wire will provide information about its temperature. Given that much more energy is deposited in heat than in secondary particles, we expect that using the wire temperature to measure the beam profile will be much more efficient than traditional techniques. The technique will be more sensitive, have higher signal to noise ratio, and will have better resolution, thus making it possible to measure the low-intensity halo and tails of the beam. The scan speed is adjusted according to the expected intensity of the beam to avoid meltdown or increase resolution.

Using the vibrating wire scanner technique, initial profile measurements were performed on laser beams $[3,4]$ and air jets [5] to estimate the resolution of the temperature rise, which was about $10^{-4}{ }^{\circ} \mathrm{C}$. Then we measured the profile of a low-intensity electron beam with an average current of a few $n A$.

\section{EXPERIMENTAL RESULTS}

\section{A. Description of experimental setup}

A string resonator was constructed using a vibrating wire $90 \mu \mathrm{m}$ in diameter. The wire is located between two samarium-cobalt permanent magnet pole pieces with opposite magnetic fields to vibrate the wire while minimizing the magnetic field effect of the electromechanic resonator on the charged particle beam [5]. A quartz bushing acts as the resonator base, and an electronic oscillator unit generates the natural oscillations of the wire in the 2 nd harmonic.

To increase the measurement accuracy within a given time interval, the frequency of the oscillator unit was multiplied by 32 in the readback unit and the signal was transferred into a custom-made computer interface board. The board has a standard RS232 port, two channels for frequency measurement, three channels for analog signal measurement, and eight digital outputs.

The stepper motor control unit and the electronic oscillator have an autonomous power supply from a battery and are located in the accelerator ring, in the immediate proximity of the scanner. All the electronics in the ring and the data lines are isolated from the accelerator ground. The operation of the scanner is controlled by a MS-DOS program, which measures the frequency of the wire oscillations according to the following algorithm. Move the wire by a set increment, wait $1 \mathrm{sec}$, and measure the frequency of the wire several times for averaging. The $1 \mathrm{sec}$ wait is more than enough time for the wire

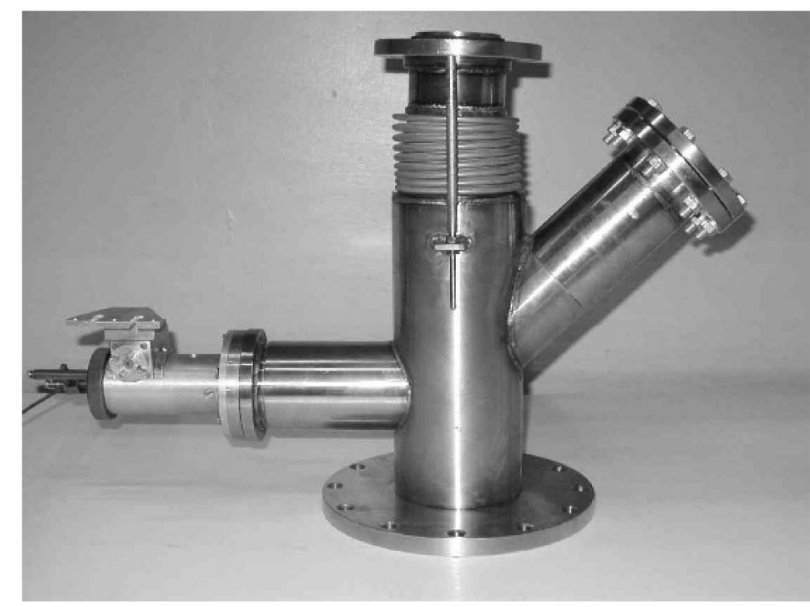

FIG. 1. The vibrating wire scanner vacuum chamber with ports for the scanner drive mechanism (left) and viewing port (right). 
temperature and as a consequence the natural vibrating frequency to come to a steady state.

\section{B. Vacuum chamber of the VWS}

To install the scanner, we made a special vacuum chamber section with a $90^{\circ}$ port for mounting the linear scanning mechanism (see Fig. 1, left port). The diagonal $45^{\circ}$ port with a window allows us to inspect the wire and also provides the flexibility to view the beam on a profile screen, which could be mounted on the same shaft as the vibrating wire. The $60 \mathrm{~mm}$ diameter beam pipe includes a bellows for alignment purposes. The operation of the scanner had no ill effect on the vacuum quality in the beam line.

\section{Scanning mechanism}

The wire diameter is $90 \mu \mathrm{m}$ and the length is $36 \mathrm{~mm}$. The scanning mechanism consists of a microscrew moved by the computer-controlled stepper motor as described above. The number of steps can vary from 1 up to 30500 . For this experiment the step size was $1 \mu \mathrm{m}$ and the travel range $32 \mathrm{~mm}$. Finer steps are possible depending on the stepper motor system. The maximum speed of the wire motion was $0.2 \mathrm{~mm} / \mathrm{sec}$. Two limit switches fix the absolute position of the scanner. The limit switch used for defining the park position allows us to find the scanner "home" position within $\pm 5 \mu \mathrm{m}$.

\section{Installation of the scanner into the accelerator beam line}

In this experiment the scanner was mounted on the injector-to-synchrotron extraction line after a collimator, where the electron beam was at $20 \mathrm{MeV}$ and $6 \mathrm{nA}$. The wire was positioned vertically and scanning was per- formed in the horizontal direction. The data line from the scanner to the control room is about $70 \mathrm{~m}$ long.

Tests of the wire oscillation mechanism show that the wire behaves the same way in the accelerator vacuum system as it did in atmospheric laboratory conditions. However, due to signal losses on the long data line from the scanner to the control panel, the frequency signal amplitude dropped below the discrimination threshold and some distortion of the signal appeared as compared to laboratory conditions. To improve signal transmission between the wire oscillation generator and the computer interface board, a sharpener amplifier was mounted on the control panel, which provided reliable frequency readings.

\section{E. Scanner runs with no beam}

The next step in the experiment was to monitor the VWS frequency without any beam and the machine off condition. We observed a symmetric noise signal with a standard deviation of about $0.3 \mathrm{~Hz}$.

Switching on the injector system and producing an electron beam but directing it to a dump before the scanner, resulted in a considerable electrical disturbance. We observed a $50 \mathrm{~Hz}$ alternating signal with an amplitude of $72 \mathrm{~V}$ between the ground of the scanner measurement station and the accelerator ground, superimposed with a $50 \mathrm{KHz}$ lower amplitude noise. We also observed a dc bias of $-1.1 \mathrm{~V}$. In addition the mean wire frequency decreased by approximately $2 \mathrm{~Hz}$ and the signal to noise ratio increased as measured in the control room (Fig. 2).

\section{F. Scanning the beam}

We made electron beam profile scan measurements after turning off the bending magnet to the dump and directing the beam to the wire scanner. We observed no

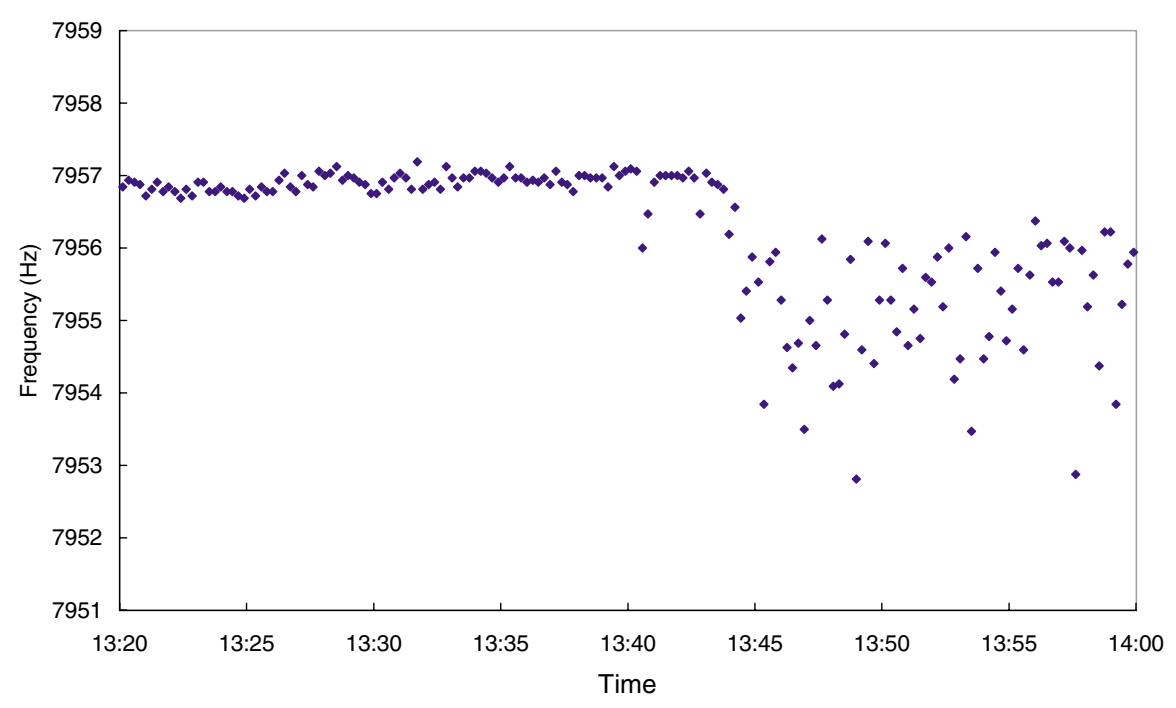

FIG. 2. (Color) Wire oscillation frequency measurement in the control room. 


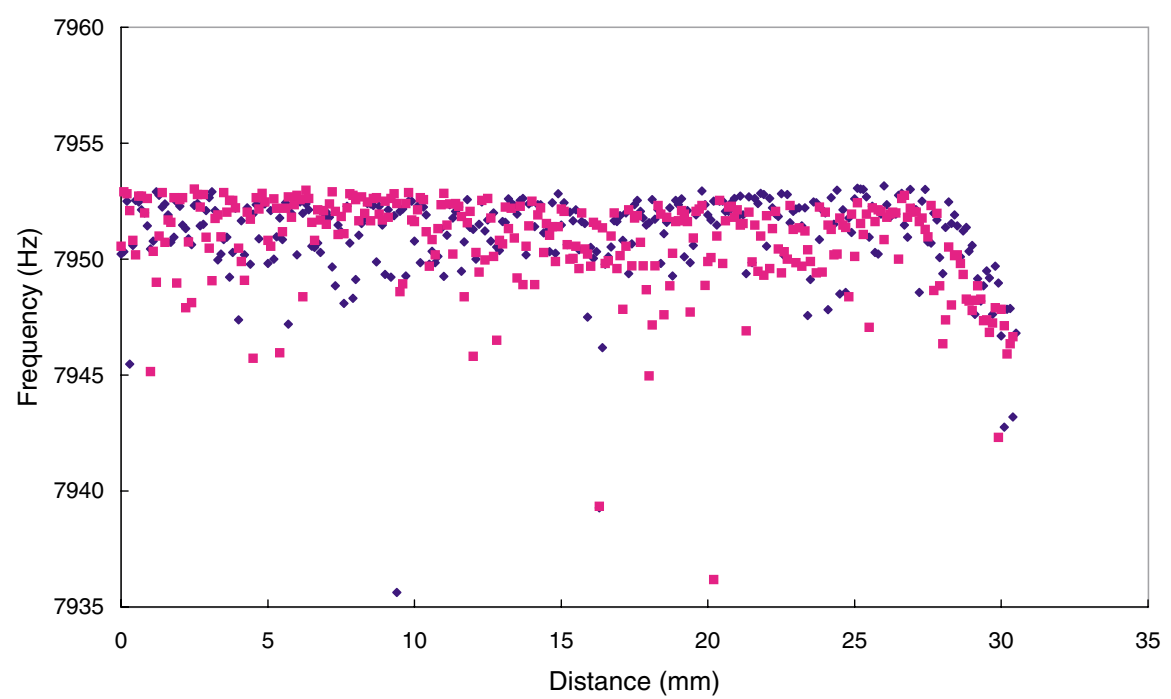

FIG. 3. (Color) Profile scan of the beam positioned in the center of the vacuum chamber. The blue diamonds correspond to the forward movement and the purple squares to the backward movement of the scanner.

change in the readback for the home position of the wire, which is far away from the beam. The beam current at the vibrating wire scanner was about $6 \mathrm{nA}$ as monitored by a Faraday cup $2 \mathrm{~m}$ downstream of the wire scanner station. The beam diameter at the phosphor screen, $40 \mathrm{~cm}$ upstream of the VWS, was about $\sigma=5 \mathrm{~mm}$.

Several full-scale beam scans were conducted. Because of the limited travel range of the scanner shaft, the scanner could not reach the beam center, thus it was impossible to scan the entire cross section of the beam. The scanning parameters were as follows: $0.1 \mathrm{~mm}$ travel at a speed of $0.2 \mathrm{~mm} / \mathrm{sec}$, a $1 \mathrm{sec}$ wait for damping the scanner vibrations, and a $1 \mathrm{sec}$ measurement gate. Figure 3 shows the frequency scan from this measurement. It can be seen how the beam heats the wire, resulting in a distinct down slope in the frequency distribution starting at about $27 \mathrm{~mm}$ of the scanner travel. One can notice a slight hysteresis in the data between the forward (blue diamonds) and backward (purple squares) travel of the scanner equivalent to a size differential of $0.7 \mathrm{~mm}$. The observed frequency differential of about $5 \mathrm{~Hz}$ corresponds to an average wire temperature change of $0.16^{\circ} \mathrm{C}$ along the wire length [4].

For scanning the full profile of the beam, it was steered towards the scanner park position and then scanned. The results of this scan are shown in Fig. 4.

As shown in Fig. 4, the frequency has a small but distinguished decrement in the interval between 21 and

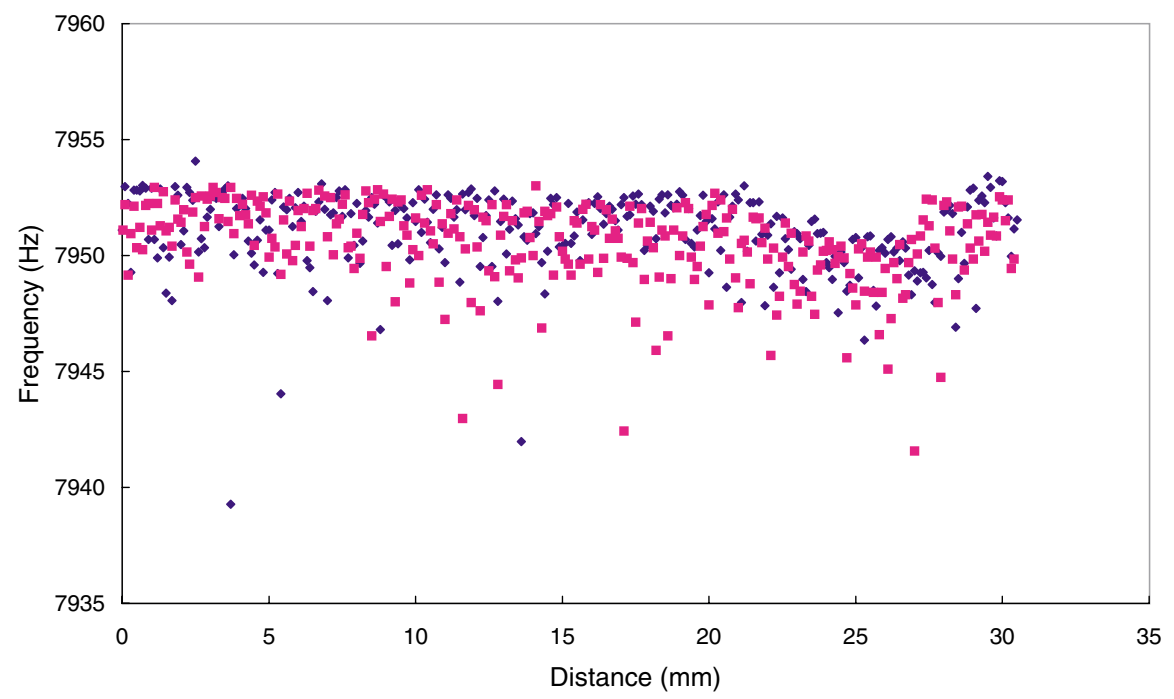

FIG. 4. (Color) Profile scan of the steered beam. The blue diamonds correspond to the forward movement and the purple squares to the backward movement of the scanner. 
$28 \mathrm{~mm}$, which is caused by the interaction between the beam and the wire. Such a frequency change corresponds to the wire heating by $0.08^{\circ} \mathrm{C}$ averaged along the wire. The depth of the profile was less than in the case for the centered beam, which may be caused by various effects due to steering the beam to the side of the pipe.

\section{BEAM PROFILE ANALYSIS}

The vertical vibrating wire was scanned in the horizontal direction. The portion of the beam intercepting the wire heats the wire. The temperature profile along the wire is a result of the thermal balance between thermal influx from electrons, proportional to the local intensity of the beam, and heat dissipation from the wire. The primary method of heat sink in vacuum is conduction along the wire. Since the wire is much longer than the transverse size of the beam, we can model the wire temperature profile along the wire as a triangle with temperature $T$ in the middle where the wire intercepts the beam and the ambient temperature $T_{0}$ at the ends. Thus the dependence of temperature $T$ on wire oscillation frequency $f$ can be expressed as

$$
T-T_{0}=-\frac{8 l^{2} \rho}{E \alpha_{S}}\left(f^{2}-f_{0}^{2}\right)
$$

where $l$ is the length of the wire, $\rho$ is the density, $E$ is the modulus of elasticity, $\alpha_{S}$ is the coefficient of thermal expansion of the wire material, and $f_{0}$ is the basic frequency of the wire oscillations without beam.

The temperature $T$ of the wire is proportional to the number of particles intercepting the wire in a given position. The proportionality factor is determined by the ionization losses of electrons in the wire. Since we know the total current of the beam, we can normalize the horizontal profile of the beam by the proportionality factor.

Figure 5 shows the result of beam profile reconstruction for the first scan in Fig. 3. The $0.7 \mathrm{~mm}$ backlash (or hysteresis) of the drive mechanism during the forward and backward scanning is taken into account. The experimental data are averaged over five points along the horizontal axis. We also discard points lower than the $2 \mathrm{~Hz}$-wide corridor from the top of the scan data shown in Figs. 3 and 4. The beam profile shown in Fig. 5 is approximated by a Gaussian function with $\sigma=1.48 \mathrm{~mm}$ and beam centroid at $30.87 \mathrm{~mm}$ from home position. The overall beam current is set at $I_{0}=6 \mathrm{nA}$. As mentioned above the VWS travel distance was limited by the length of the shaft and we could scan only half of the beam.

Figure 6 shows a similar horizontal beam profile for the second frequency scan shown in Fig. 4. Comparing the signals from the two scans, and knowing that the beam current was $6 \mathrm{nA}$ in the first scan, we deduce that shifting of the beam to the side of the beam pipe reduced the total current to $3.4 \mathrm{nA}$. It also shows a slight beam profile asymmetry, which is to be expected when the beam is not centered in the quad lattice. As for the first case, these data were also approximated by a symmetric Gaussian function. The standard deviation $\sigma=1.98 \mathrm{~mm}$ and beam central position was at $25.4 \mathrm{~mm}$ from the wire home position.

At a distance of $1.48 \mathrm{~mm}$ from the beam centroid, the vibrating wire scanner intercepts $69 \mathrm{pA}$ of beam current and produces a very reliable signal. The current density on the wire here is $0.8 \mathrm{pA} / \mu \mathrm{m}$. As an example, the vibrating wire scanner can be very useful for the TTF FEL. The current traditional wire scanner planned for the TTF FEL requires an intercepting current density of at least $5 \mathrm{nA} / \mu \mathrm{m}$ at the $3 \sigma$ from the beam centroid [6]. The lower intercepting current density required by the VWS

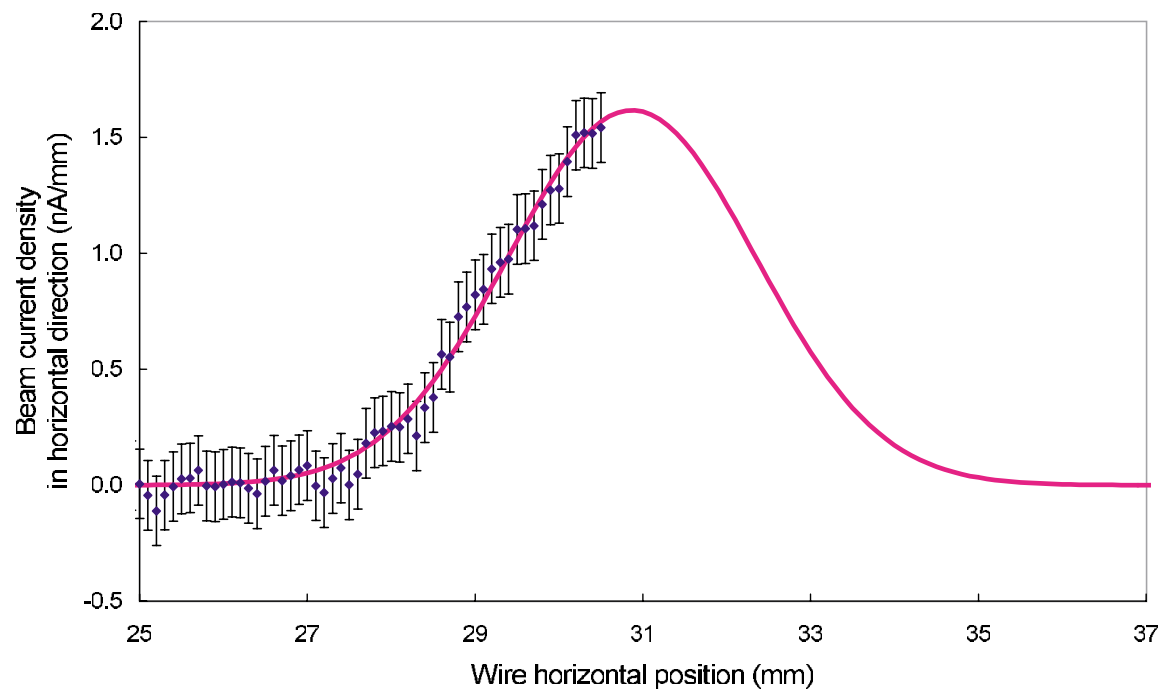

FIG. 5. (Color) Horizontal profile of the $6 \mathrm{nA}$ beam reconstructed from the frequency scan in Fig. 3. 


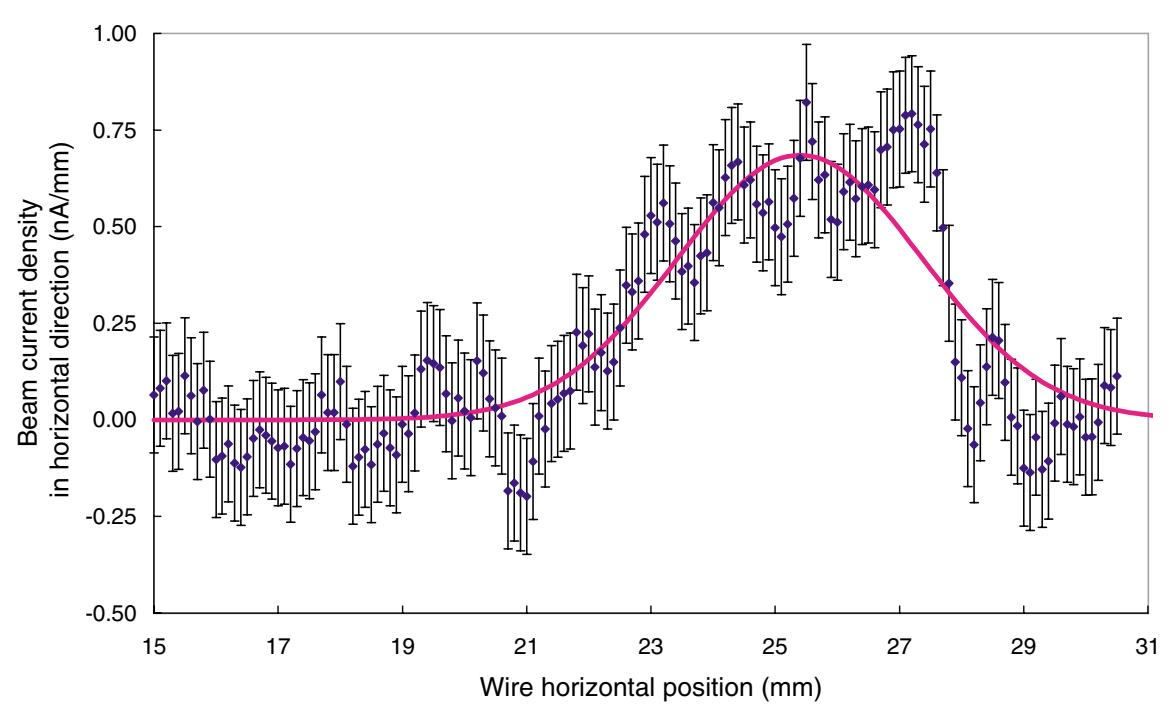

FIG. 6. (Color) Horizontal profile of the beam which was steered towards the side of the beam pipe.

will allow for a better profile resolution on the TTF FEL beam transverse profile and tail measurements.

\section{CONCLUSION}

These proof of principle experiments confirmed our expectation on the possibility of using a VWS for charged particle beam transverse profile measurements. These measurements are particularly useful for low current beams. Specifically, it was confirmed that (i) interaction of the beam with the wire does not quench the wire oscillations; (ii) the change in the natural oscillation frequency of the wire during interaction with the beam is a reliable method of measuring the transverse profile of extremely low-intensity beams; and (iii) the vibrating wire scanner can be a valuable tool for measuring lowintensity beam profiles, beam halo, and tails.

In these experiments the accelerator $\mathrm{rf}$ system induced noise in the measurement system, which can be solved by standard engineering techniques and indeed we have solved it for subsequent experiments.

\section{ACKNOWLEDGMENTS}

This work was sponsored by ISTC Project No. A-347 and the Republic of Armenia theme No. 1142. The authors would like to thank R. O. Avakian, A. E. Avetissian, and S. M. Darbinian for the use of the Yerevan Physics Institute accelerator injector and for profitable discus- sions. We also thank A. D. Yeremian for numerous helpful comments.

[1] S. G. Arutunian, N. M. Dobrovolski, M. R. Mailian, I. G. Sinenko, and I. E. Vasiniuk, Phys. Rev. ST Accel. Beams 2, 122801 (1999).

[2] P. Elmfors, A. Fasso, M. Huhtinen, M. Lindroos, J. Olsfors, and U. Raich, Nucl. Instrum. Methods Phys. Res., Sect. A 396, 13-22 (1997).

[3] S. G. Arutunian, N. M. Dobrovolski, M. R. Mailian, V. A. Oganessian, and I. E. Vasiniuk, in Proceedings of the Conference Laser 2000, Ashtarak, Armenia (Publishing House of NAS of Armenia, Ashtarak, 2000), pp. 81-84.

[4] S. G. Arutunian, N. M. Dobrovolski, M. R. Mailian, V. A. Oganessian, and I. E. Vasiniuk, in Proceedings of the NATO Advanced Research Workshop on Electron-Photon Interaction in Dense Media, Nor Amberd, Armenia, 2001, NATO Science Ser. II, Vol. 49, pp. 303-308.

[5] S. G. Arutunian, A. E. Avetisyan, N. M. Dobrovolski, M. R. Mailian, I. E. Vasiniuk, K. Wittenburg, and R. Reetz, in Proceedings of the 8th European Particle Accelerator Conference, Paris, France, 2002 (EPS-IGA/ CERN, Geneva, 2002), pp. 1837-1839 (http://accelconf.web.cern.ch/AccelConf/e02/PAPERS/THPRI054.pdf).

[6] S. Striganov, G. Schmidt, and K. Wittenburg, Estimation of the signal from the wire scanner in the TTF, desyntwww.desy.de/mdi/downloads/WIRE_TTF.pdf 